\title{
INVESTIGATION OF THE WEAR BEHAVIOR OF THE SLIPPER IN AN AXIAL PISTON PUMP BY MEANS OF SIMULATION AND MEASUREMENT
}

\author{
Roman Ivantysyn*, Ahmed Shorbagy, Jürgen Weber \\ Institute for Fluid-mechatronic Systems, Technische Universität Dresden, Helmholtzstrasse 7a, 01069 Dresden \\ * Corresponding author: Tel.: +49 351 46333701; E-mail address: roman.ivantysyn@tu-dresden.de
}

\begin{abstract}
Axial piston pumps are universal displacement machines that are used in a vast variety of applications. Their high pressure resistance and ease of operation make them very popular, especially in mobile applications. Some applications require more robust pumps with an extended lifetime, particularly those that operate in remote environments such as marine type or mining operations. Especially new applications like displacement control have high demands on pumps such as through shaft operation (many pumps on one shaft), high dynamics and multi-quadrant operation. These demands create challenges in terms of lifetime expectancy and robustness for pump manufacturers and machine OEMs. Currently most axial piston pumps go through a run-in process. During this process the softer bronze parts shave off and change their shape according to the necessary one for the pumps' proper operation. This process is highly dependent on the design of the parts and their manufacturing tolerances. In this paper the run-in process of the slippers of an axial piston pump was investigated by means of measurements of the gap height and wear profile as well as simulation. The measurements show a clear change of profile and gap heights for the first $120 \mathrm{~h}$ of the pumps operation. After that the gaps stabilize. The numerical simulations made with the program Caspar FSTI were coupled with contact wear models to output wear profiles. Different models will be introduced and compared with measurements. Both the amount of material removed and the performance of the pump before and after run-in will be discussed.
\end{abstract}

Keywords: Axial Piston Pumps, Wear, Gap Measurement, Simulation

\section{MOTIVATION}

Due to their high energy density, high efficiency and overall robustness, axial piston machines have been the primary choice for high pressure and mobile applications. The lifetime of such a machine can be very high, especially due to their hydrostatic bearings. However, todays pumps and motors go through a critical run-in process, where the nominal machined parts wear in. This wear can produce particles that influence other components downstream of the system. It is primarily the sealing lands that go through this crucial process, which are already machined within micron tolerances, changing their surface topology in order to withstand the required loads.

The moving parts of an axial piston pump and illustrations of the fluid films separating the parts, are shown in Figure 1. The slipper/swash plate interface, being the focus of this investigation, is shown on the right hand side.

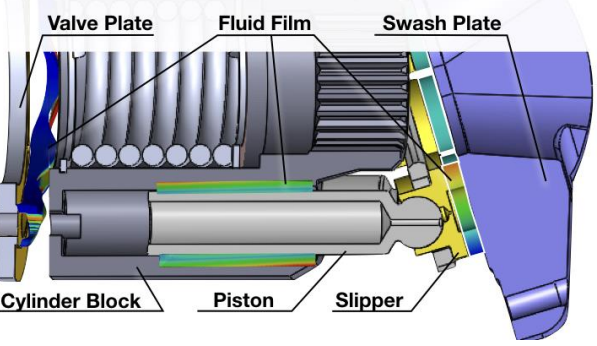

Figure 1: Tribological interfaces in axial piston pumps and their fluid films

The fluid film between the slipper and swash plate is a highly complex one, as it ranges from very small gap heights $(<5 \mu \mathrm{m})$ on the high 
pressure side, to rather large gap heights (around $100 \mu \mathrm{m}$ ) on the low pressure side, at typical fluid film behavior is shown in Figure 2. The simulation model was verified through very extensive measurements on two different pump designs, since the gap height ranges vary for each design $[1,2]$.

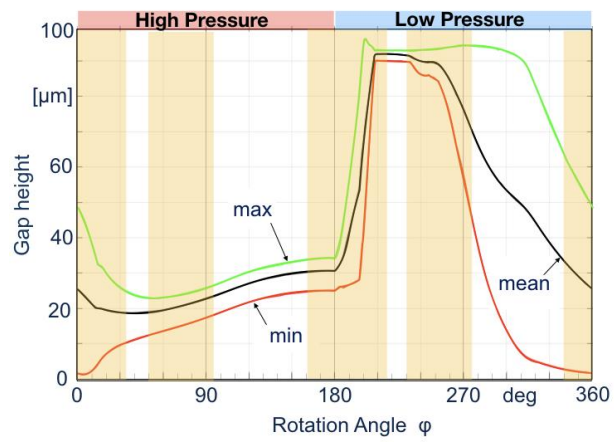

Figure 2: Typical Slipper Gap Heights of an Open Circuit Pump, simulated with Caspar FSTI and validated at the Highlighted Sections

Measurements published in a previous paper revealed that the slippers go through a run-in process, which can take up to 100 operating hours [1]. During this run-in the parts change their shape and have been shown to perform worse after the run-in was completed compared to before for this particular pump design. Other findings revealed that for specific operating conditions the simulated temperature field only matched the measurement, if the resulting material removal due to wear was considered in the simulation [3]. This wear process will be studied more in detail in this paper.

\section{RESEARCH GOAL AND APPROACH}

The aim of this paper is to present an approach to simulate the slipper run-in at various operating points using Caspar FSTI, which is a simulation tool that is capable of calculating the gap heights in axial piston pumps using numerical methods. The tool is explained in detail in $[2,4,5]$, where the slipper calculations are described in full detail in Schenk [2].

The current version of Caspar FSTI does not incorporate the calculation of the wear of the parts. However, it is possible to input a measured wear profile, which enables indirect wear analysis. This is useful if a part design already exists.
The goal of the authors research is to develop a method that is capable of predicting the resulting wear based on simulation results only. Pump designers can then use this method to design parts with less, or possible even no wear. An example for this could be micro-surface shapes, which have shown to reduce the run-in of parts while also increasing efficiency [6]

\section{GAP HEIGHT MEASUREMENTS}

A $92 \mathrm{cc}$ open circuit pump was analyzed for this research. A series of measurements were performed, including temperature and gap height measurements at both the valve plate and the swash plate of the pump. The methods, measurements and results can be seen in full detail in $[1,3,6,7,8]$. Both the valve plate as well as the slippers, each being the softer material than their counterpart glide partner, went through a run-in process, which could be observed in the measured signals.

The slipper gap height measurements were particularly interesting as they clearly illustrated the change in the fluid film thickness and the motion profile of the slipper throughout the runin process. The fluid film thickness was measured using three eddy current sensors, carefully placed at four positions total, high and low pressure side, bottom dead center (BDC) as well as top dead center (TDC). The sensor placement at TDC is shown in Figure 3. The three sensors are positioned in a fashion, which enables an exact measurement at the $\operatorname{TDC}\left(\operatorname{phi} \varphi=180^{\circ}\right)$.

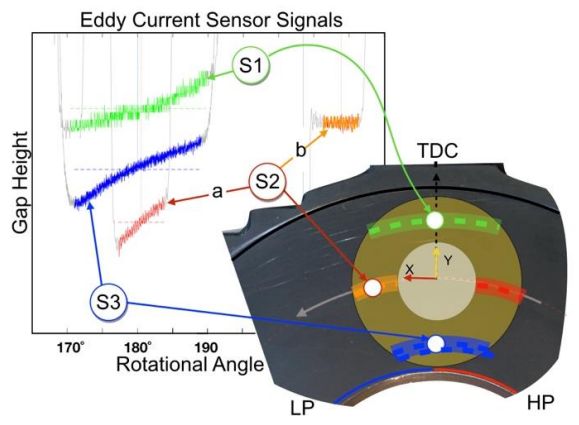

Figure 3: Measurement Location of the Eddy Current Sensors at the TDC

The graph in Figure 3 illustrates the individual sensor outputs as the slipper slides over the TDC. It can be seen that sensors 1 (S1 in green) and 3 (S3 in blue) deliver a reading for the outer and 
inner fluid film, respectively. Sensor 2 (S2a in red and $\mathrm{S} 2 \mathrm{~b}$ in orange) outputs two narrow bands as its reading is interrupted by the pocket. If $\mathrm{S} 1$ is higher than S3 the slipper is tilting towards the inside (around the $\mathrm{x}$ axis). If S2a is lower than $\mathrm{S} 2 \mathrm{~b}$, then this indicates either that the slipper is lifting off after the TDC or that slipper is tilted forward (around the y-axis). The individual sensor signals also entail information about the current movement of the slipper at that instance in time. These individual slipper measurements (S1-3) were averaged over 50 revolutions for each slipper. The results for one operating point are shown in Figure 4 and 5. Since the pump consists of multiple individual slippers (nine in this case), it was observed that each slipper has a different initial gap height (Figure 4), and performs an individual run-in process. The measured fluid film thicknesses are shown for each slipper at the initial run ( $\mathrm{t}=0 \mathrm{~h}$ in Figure 4) and after $120 \mathrm{~h}$ (Figure 5)

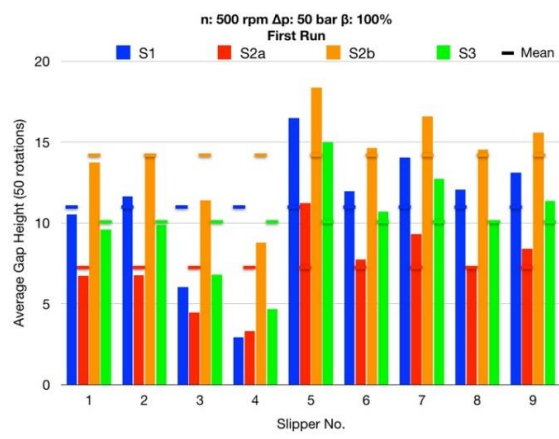

Figure 4: Measured Slipper Gap Height at $\mathrm{t}=0 \mathrm{~h}$

The horizontal lines that cross those bars are the mean of all slippers and act as a reference how uniform the slippers are to each other. For the initial run, it can be seen that many slippers deviate from the mean, some quite significantly. After the run-in the slippers are all very close to the mean.

In Figure 4 each of the 9 slippers has a different gap height and position in space, as show in the relation between the sensors to each other. For example, in slipper 1 the S1 signal is higher than S3, indicating that the slipper is tilted slightly towards the inside. The mean gap height is $9 \mu \mathrm{m}$. However, slipper 3 shows an opposite tilt and an average gap height of $6 \mu \mathrm{m}$. Same analysis can be made for all 9 slippers.

After 120 operating hours (Figure 5) the gaps have all converged to a uniform fluid film. After the initial measurements slipper 5 was replaced with a different shape to act as a trigger, therefore it is missing, however all other 8 slippers are very close to the mean and show the same trends as well as positions in space.

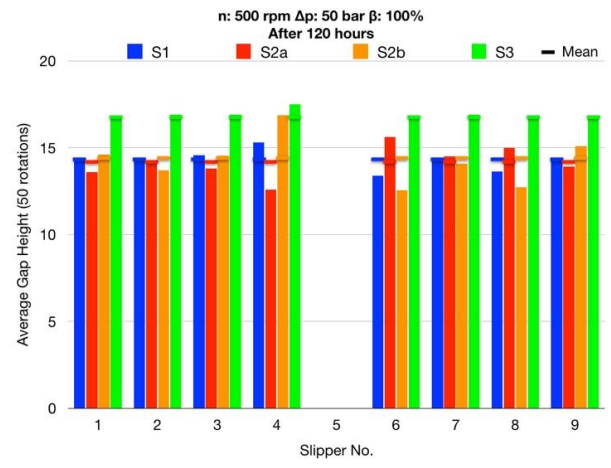

Figure 5: Measured Slipper Gap Height at $\mathrm{t}=120 \mathrm{~h}$

It is important to note, that the pump was measured in a total of 78 different operating points from the initial measurement and the final after 120 hours. This means that the pump was run-in at various operating points and not just one single one. This would reflect a typical real-world application.

Interestingly the gap height of all slippers is higher after the run-in, than compared to the initial non-worn measurement. Higher gaps mean less viscous friction, but higher leakage. For each operating condition there is an optimal gap height, meaning the sum of the losses due to friction and leakage are at a minimum. The losses can be calculated as follows:

$P_{\text {leak }}=Q_{S G} p_{G}=\frac{\pi h_{G}^{3} p_{G}^{2}}{6 \mu \ln \left(\frac{r_{\text {out }}}{r_{\text {in }}}\right)}$

$P_{\text {friction }}=F_{T G} \omega R_{B}=\pi\left(r_{\text {out }}^{2}-r_{\text {in }}^{2}\right) \mu \frac{\omega^{2} R_{B}^{2}}{h_{G}}$

where $\mathrm{h}_{\mathrm{G}}$ is the gap height, $\mathrm{p}_{\mathrm{G}}$ is the pressure in the pocket, $\omega$ is the rotational speed, $\mu$ is the dynamic viscosity and $r_{\text {out }}$ and $r_{\text {in }}$ represent the inner and outer diameter of a single land slipper. The power loss is the sum of the $\mathrm{P}_{\text {leak }}$ and $\mathrm{P}_{\text {friction. }}$ The gap height is assumed to be constant and uniform for these analytical calculations [10].

The power losses for different slipper gap heights at $500 \mathrm{rpm}, 50 \mathrm{bar}$ and $100 \%$ displacement, the same operating condition as the measurement in Figure 4-5, is shown in Figure 6. Here the viscous and leakage losses are plotted with varying slipper gap height. 
It can be observed that small changes in fluid film thickness can lead to rather large changes in power loss ( $10 \mathrm{~W}$ equal $2.6 \%$ efficiency drop).

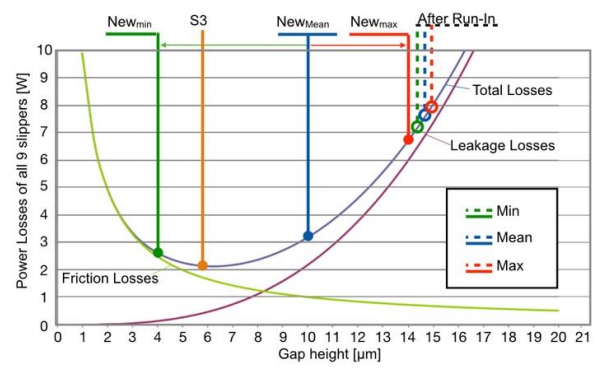

Figure 6: Predicted Power Losses with Changing Gap Heights at $500 \mathrm{rpm} 50$ bar 100\% Disp.

In Figure 6 the measured gap heights of the 9 slippers in their new shape $(\mathrm{t}=0 \mathrm{~h}$ in solid lines $)$ and after the $120 \mathrm{~h}$ of operation (after run-in in broken lines) are plotted. The slippers in their new condition have a large spread in gap height, ranging from 4 to $14 \mu \mathrm{m}$ resulting in power losses ranging from $2-6.5 \mathrm{~W}(0.5-1.7 \%$ of the total power output) for all slippers. Slipper 3 had a rather perfect shape for this operating point, resulting in the lowest possible power loss at just $2 \mathrm{~W}$.

After the run-in process the slippers all have converged to a tighter spread in gap heights, suggesting that they all have uniform surface shapes post run-in. However, the resulting fluid film height was higher than the worst of the slippers before the run-in $(14.2-15.2 \mu \mathrm{m})$ and resulting in even worse power losses around $8 \mathrm{~W}$ which equals $2 \%$ efficiency drop of the pump.

This trend could be observed in a multitude of operating conditions, one other example can be seen in Figure 7 for a higher speed and higher pressure. Here the power losses are an order of magnitude higher, resulting in a difference of $50 \mathrm{~W}$, which results in a $2 \%$ total efficiency drop at this operating point between the best new design and the worst worn in design for the entire pump.

These results clearly show that the run-in process must not result in an optimal shape of the parts, but rather serves the purpose to smoothen out manufacturing tolerances. The resulting shape can perform worse than before the run-in process. These findings cannot be taken as a general trend, as this was only measured on one pump series. However, they show that the run-in process needs to be considered during the design process, as it can influence the efficiency of the parts drastically.

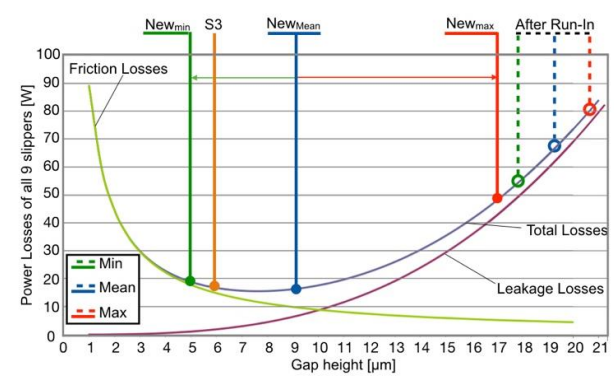

Figure 7: Predicted Power Losses with Changing Gap Heights at $1500 \mathrm{rpm} 100$ bar 100\% Disp.

\section{WEAR PREDICTION MODEL}

Simulation tools are becoming more and more important in today's design process. CAD, CFD and FEM tools are one example how software tools have enabled new designs and developments by giving engineers a better insight into the products they are developing. The development of a capable tool to simulate the performance of axial piston pumps has been the goal of many researchers $[12,12,13]$. Such a tool would enable the use of new materials, shapes and most importantly optimization of the sealing lands of these machines. Caspar FSTI was developed by a series of researchers throughout many years and publications $[2,4,5,9]$. This tool is already capable of simulating and analyzing the fluid film in axial piston pumps and has been validated in many different pumps and motors [1, $2,3,4,5]$. However, the wear-in process has not been fully captured by this tool yet. Therefore, the goal of this research is to develop a wear tool that uses Caspar FSTI to predict the wear-in process and more importantly the resulting performance of the parts after such wear.

The simulation solves a simplified version of the Navier-Stokes equation as shown in Figure 8. The simplifications are 1) no transient flow is considered in the gap 2) no fluid inertia is considered, 3) fluid forces are neglected. The resulting equation is further simplified assuming laminar flow, and no changes in velocity along $\mathrm{x}$ and $\mathrm{y}$ in the fluid grid (refer to Figure 9 top left). The resulting equation is then solved along with the energy equation, Newtons equation of motion and considering deformation due to pressure and temperature. The thermal 
temperature loop, which calculates the temperature of the parts and the fluid, is updated after each simulated revolution is completed, using the viscous losses as the heat source. The simulation is seen as completed when the temperature of the parts converged to a steady state value.

$$
\left.\begin{array}{l}
\nabla p=\nabla \cdot(\mu \nabla v) \\
\frac{\partial p}{\partial x}=\mu\left(\frac{\partial^{2} u}{\partial x^{2}}+\frac{\partial^{2} u}{\partial y^{2}}+\frac{\partial^{2} u}{\partial z^{2}}\right) \\
\frac{\partial p}{\partial y}=\mu\left(\frac{\partial^{2} v}{\partial x^{2}}+\frac{\partial^{2} v}{\partial y^{2}}+\frac{\partial^{2} v}{\partial z^{2}}\right) \\
\frac{\partial p}{\partial z}=\mu\left(\frac{\partial^{2} w}{\partial x^{2}}+\frac{\partial^{2} w}{\partial y^{2}}+\frac{\partial^{2} w}{\partial z^{2}}\right.
\end{array}\right\} \begin{aligned}
& \frac{\partial p}{\partial r}=\mu\left(\frac{\partial^{2} v_{r}}{\partial z^{2}}\right) \\
& \frac{1}{r} \frac{\partial p}{\partial \theta}=\mu\left(\frac{\partial^{2} v_{\theta}}{\partial z^{2}}\right) \\
& 0=\mu\left(\frac{\partial^{2} w}{\partial z^{2}}\right)
\end{aligned}
$$

Figure 8: Simplifications of the Navier-Stokes Equation in Caspar FSTI

The primarily outputs of the simulation are the gap heights, temperature and pressure fields at each fluid grid point for each time step. They are illustrated in Figure 9 for the slipper / swash plate interface.
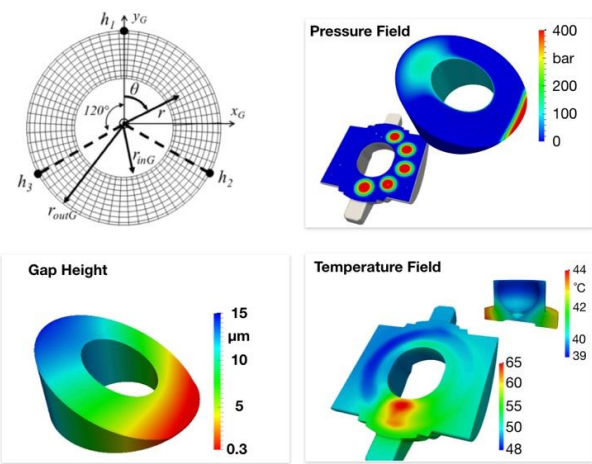

Figure 9: Fluid grid (top left), and important simulation outputs: gap height (bottom left), pressure and temperature field of the slipper during a wear process (right).

Other significant outputs of the simulations are the power losses, viscous friction, leakage, thermal load and contact pressure. The contact pressure is particularly interesting in this case, it is only outputted if the simulation is unable to balance the forces acting on the slipper with the pressure in the fluid film. Meaning that the fluid film alone is not sufficient to bear the loads and in reality it would come to metal to metal contact between the moving parts. The contact pressure is therefore the difference in the maximum pressure the fluid film is able to exert and the acting pressure from the external forces at one grid point. An example of a contact pressure is presented in Figure 10. Here the fluid film is shown as a function of rotation angle for a medium speed medium pressure operating point. The minimum gap height is plotted in blue. It can be observed, that the gap is very small around the TDC ( $\phi=180^{\circ}$, Figure 10 top left). For this instance in time, TDC at this operating point, the resulting pressure field, gap height and temperature of the swash plate can be seen in Figure 9. The pressure is extremely high, $4 x$ the pressure in the displacement chamber. The corresponding gap height is $0.3 \mu \mathrm{m}$ at the leading edge of the slipper. This is the smallest possible gap height, as the surface roughness is larger than that. Therefore, the simulation will not decrease the gap further, but rather will counter the loading force with a contact pressure. The distribution of the corresponding contact pressure can be seen in Figure 10 top right. The resulting normal force is the contact pressure multiplied with the area of the fluid cell - its distribution is visualized on the bottom left. Finally, the contact forces that occur at each grid point during one revolution are added, the result is plotted on the bottom of the figure. This force will now be used in the wear model.
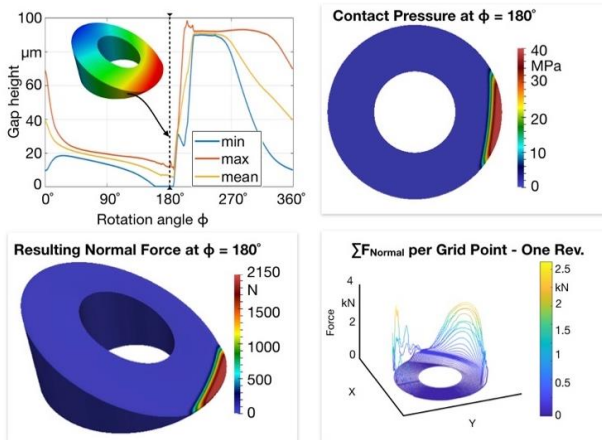

Figure 10: Contact Force Generation; min. Gap Height (top left), Resulting Contact Pressure at min. Gap Height (top right), Resulting Normal Force at min. Gap Height (bottom left), Summed Up Normal Force for one Revolution at each Grid Point (bottom right). 


\subsection{Archard's Wear Model}

Archard developed a simple wear model, which is based on equation (3) below, where $\mathrm{S}$ is the contact length, $\mathrm{N}$ the normal force, $\mathrm{H}$ the hardness of the material and $\mathrm{K}$ is a wear coefficient.

$W=K \frac{S N}{H}$

The wear coefficient is therefore the only unknown in this approach. There are multiple publications that studied this coefficient, Romalho and Miranda for example have discovered that a linear correlation as suggested by Archard, can be confirmed by measurements [14]. Amiri used an energy-based approach, which seems very intuitive and most importantly physics based $[15,16]$. In his work the coefficient $\mathrm{K}$ is based on the slope of the measured temperature rise vs. the heat transferred to the body during a wear process between two materials, an example is shown in Figure 11. For the material pairing of the slipper and the swash plate, the slope of the curve is $\mathrm{K}=2 \times 10^{-4}$ as can be found in his work [15]. This value was used for the later explained wear model implementation into the gap simulation.

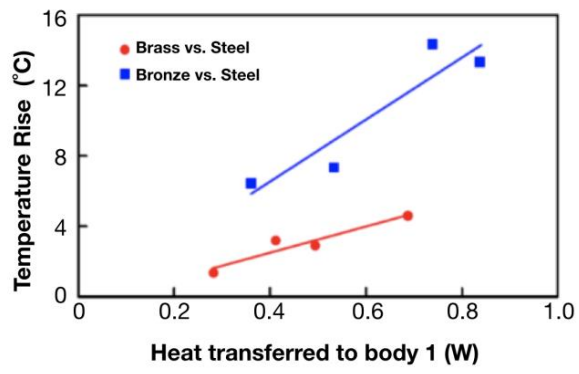

Figure 11: Experimental Determination of the Wear Coefficient [15]

Using an energy-based coefficient makes sense as the wear process must be explainable with energy conservation. Energy due to friction is converted into heat, however some energy must be lost when atoms are being broken out of their bonding. Archards's approach was first implemented into the wear model and it yielded good results as will be seen in the next chapter. However, Archard does not consider surface roughness, surface topology and other material properties that surely have an influence on the wear of the material. A more expansive approach, which considers many more factors than Archard, is discussed next.

\subsection{Fleischer's Wear Model}

Fleischer published a series of publications in German language, where he covered wear in great detail [17]. Without getting in too great of detail, his work can be summarized as follows: The wear volume $V_{V}$ is a function of the wear work - $\mathrm{w}_{\mathrm{R}}$ and the wear energy density $-\mathrm{e}_{\mathrm{R}}{ }_{\mathrm{R}}$ as shown in equation 4 .

$V_{V}=\frac{w_{R}}{e_{R}^{*}}$

To explain the wear work and energy density more information is necessary. Fleischer's work assumes that the process of wear is a series of discrete micro contacts, which are finite and mainly dependent on the surface topology. It can be described as a meshing of many tiny teeth that need form a small mechanical bond. Friction is the process that breaks this bond either by deformation in either plastic or elastic fashion, or shearing the teeth off. The smoother the surface the smaller the teeth and the less energy is required to "un-mesh" the teeth. Since the process occurs in a micro to nano scale, chemical reactions cannot be neglected, as heat and mechanical stress can lead to changes in the chemical bonds of the material, changing its material properties. This can be observed in brass materials that contain lead. They change their surface topology after the wear in process and become smoother. Chemical analyses of worn in surfaces have shown new types of alloys that were not present before the wear [18]. The wear work is the sum of all discrete miniature "unmeshing" processes and is a factor of surface roughness, material hardness, density, elasticity, chemical composition, thermal capacity, number of peaks and valleys in the surface and many more material properties of both surfaces. The wear energy density is a function of number of contacts ("teeth"), the shear rate, the shear energy, and the energy accumulation number, one of many factors that Fleischer introduced, can only be empirically determined through experimental surface analysis.

As can be seen Fleischer's approach is far more complicated and would require many more pages to explain in full detail, however it is explained in great extend in [17]. Due to the complexity of the model, especially with 
parameterizing the correct inputs, the model has not yet been fully implemented, but will be so in future work.

\section{SIMULATION RESULTS}

The following simulation results are all based on Archard's approach. Its implementation into the simulation was done in a recursive loop until the wear-in process was completed, meaning no more contact was present.

The first step in the wear simulation, is to find out which operating conditions cause the most wear for a given pump design. This can be done by measurements using particle sensors, or by a validated simulation model. Particle measurements only deliver the total wear and cannot be separated into the individual components. The simulation model for the $92 \mathrm{cc}$ pump was already verified using gap and temperature measurements in previous publications $[3,5,6]$, hence it was used to determine the most critical conditions for the slipper.

Table 1: Simulated operating conditions with contact pressure for the 92 cc pump

\begin{tabular}{|c|c|c|c|}
\hline $\mathrm{OC}$ & $\begin{array}{l}\text { Speed } \\
{[\mathrm{rpm}]}\end{array}$ & $\begin{array}{l}\text { Pressure } \\
\text { [bar] }\end{array}$ & $\begin{array}{l}\text { Displacement } \\
{[\%]}\end{array}$ \\
\hline 3 & \multirow{8}{*}{500} & \multirow{2}{*}{50} & 50 \\
\hline 4 & & & 100 \\
\hline 6 & & \multirow{2}{*}{100} & 50 \\
\hline 7 & & & 100 \\
\hline 9 & & \multirow{2}{*}{200} & 50 \\
\hline 10 & & & 100 \\
\hline 12 & & \multirow{2}{*}{300} & 50 \\
\hline 13 & & & 100 \\
\hline 16 & \multirow{6}{*}{750} & \multirow{2}{*}{50} & 50 \\
\hline 17 & & & 100 \\
\hline 22 & & 100 & 100 \\
\hline 23 & & 200 & 100 \\
\hline 25 & & \multirow{2}{*}{300} & 50 \\
\hline 26 & & & 100 \\
\hline 35 & \multirow{4}{*}{1000} & \multirow{2}{*}{200} & 50 \\
\hline 36 & & & 100 \\
\hline 38 & & \multirow{2}{*}{300} & 50 \\
\hline 39 & & & 100 \\
\hline 51 & \multirow{2}{*}{1250} & \multirow{2}{*}{300} & 50 \\
\hline 52 & & & 100 \\
\hline
\end{tabular}

All 78 measured operating conditions were simulated, they ranged from $500-1800 \mathrm{rpm}$, $50-300$ bar and $50-100 \%$ displacement. The operating conditions where the simulation predicted wear for the slipper are shown in Table 1 below. The yellow highlighted operating conditions (OC) were chosen for the wear in process, as the simulation predicted stronger wear. After the most critical operating conditions for the run-in were determined, a run-in procedure was performed for each operating point. This was done as explained in Figure 12 and $\mathbf{1 3 .}$

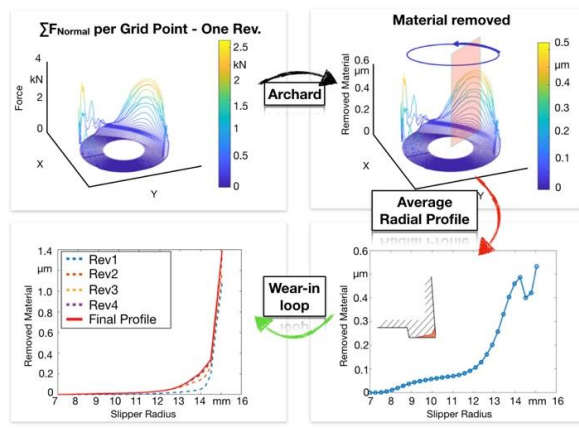

Figure 12: Wear-in profile calculation

First the non-worn surface was simulated. After the simulation converged (the part body temperature stabilized), the last simulated revolution would then be analyzed for wear. If there was contact pressure throughout the last revolution at any point, the wear was calculated using Archard's approach using the wear coefficient as described in section 4.1. The necessary normal force acting at each grid point in the gap was derived from the contact pressure. The resulting 3D wear (top right in Figure 12) was averaged around the perimeter, to yield a uniform wear profile. In reality this uniform wear takes many operating hours, as the slipper rotates around its own axis and would wear-in one small area at a time.

The averaged profile would be subtracted from the slipper material (bottom right in Figure 12) and would set the starting shape for the next simulation iteration. If the following simulation predicts contact pressure, the wear-in loop would be repeated and the resulting wear profile would be added to the previous one. This iteration was repeated until no more wear was predicted by the simulation. The wear profile would usually be quite strong in the first view iterations. For each operating point it was observed, that after the first 2 - 3 iterations, the predicted contact diminished, resulting in reduced wear as 
illustrated in the bottom left in Figure 12. The described iterative procedure, as presented in Figure 12, was repeated for all 11 operating points, as highlighted in Table 1. The results can be seen in the next section.

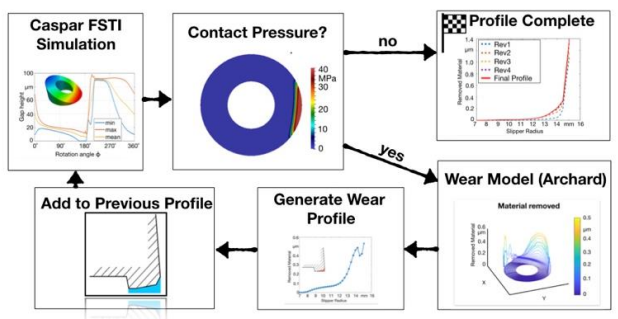

Figure 13: The Work Flow of the Wear-in loop

\subsection{Isolated operating condition wear}

In this section wear-in results are shown for various operating conditions, as predicted by the simulation using Archard's wear approach. Figure 14 shows the final wear-in profile for all 11 simulated operating conditions. It can be seen that the OC 10 and OC 22, show the most wear. Both of these operating points are at medium pressure, 200 bar, and rather low speeds.

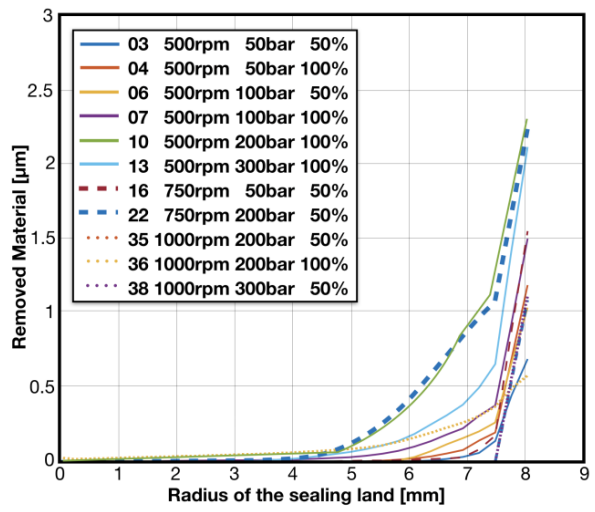

Figure 14: Final Wear Profile as Predicted by the Simulation for Various Operating Conditions.

It doesn't seem very intuitive, that the most wear occurs during a medium pressure level. One would expect it to either occur at low pressures, where the hydrodynamics of the slipper play a larger role, increasing tilt, or during high pressure, where pressure deformations tend to change the gap shape. However, it turns out that for this slipper design, the balance of the hydrostatics was quite low. This means that the hydrodynamics are needed to balance the forces, as the hydrostatics are not sufficient. At low speeds the hydrodynamics are insufficient, therefore the wear increases. As the speed increases there is barely any wear, and post $1500 \mathrm{rpm}$ there is no wear on the slippers whatsoever at any pressure level.

The impact of speed on the wear amount is shown in Figure 15. Here 4 operating points are shown. Three speed level: 500, 750 and $1000 \mathrm{rpm}$, as well as two displacement level all at the same pressure. Comparable operating points are plotted in the same color, whereas the speed levels are shown in different line types: $500 \mathrm{rpm}$ in dashed lines, $750 \mathrm{rpm}$ in dotted lines and $1000 \mathrm{rpm}$ in full lines.

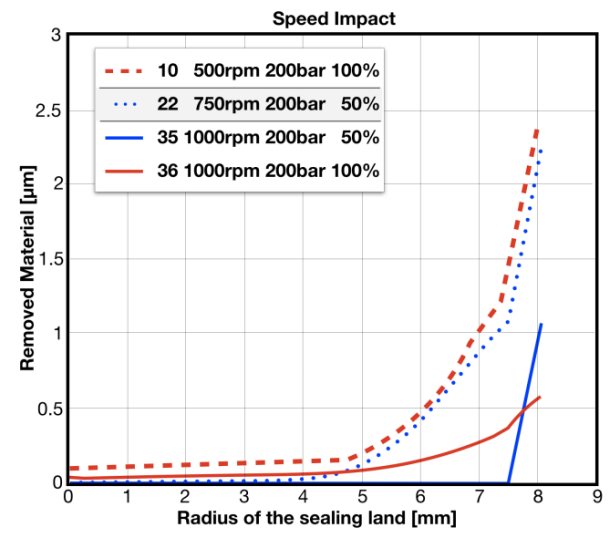

Figure 15: Speed Impact on the Wear Profile

It can be observed that lower operating speeds produce more wear than higher ones for the same pressure level. Whereas the higher the swash plate angle the higher the wear. This makes sense as the forces on the slipper increase with higher angle. This trend is displayed for more pressure levels in Figure 16. Similar to Figure 15 each displacement level has its own line shape and comparable operating points are shown in the same color at the same speed $500 \mathrm{rpm}$. It can be seen that the same trend as in Figure 15 for $1000 \mathrm{rpm}$ and 200 bar can be observed for 50 and 100 bar at $500 \mathrm{rpm}$. The lower the displacement the lower the wear.

The impact the pressure has on the wear profile is introduced in Figure 17. Four pressure level are shown at the same speed and displacement. There is a trend visible that the higher the pressure the higher the wear. This 
seems logical as the forces on the slipper increase with higher pressure, especially for underbalanced slippers. However, $200 \mathrm{bar}$ is predicated to have a higher wear than 300 bar. This seems rather counter-intuitive at first, however it does make sense as the pressure and temperature deformation of the slipper increase with higher pressure and the deformation actually increases the gap heights at the outer diameter. This also explains why there are less 300 bar operating conditions with higher wear than ones at 200 bar.

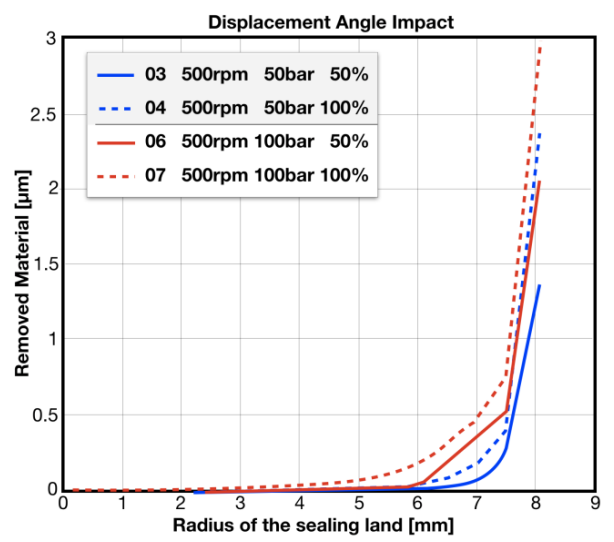

Figure 16: Impact of the Displacement Angle on the Wear Profile

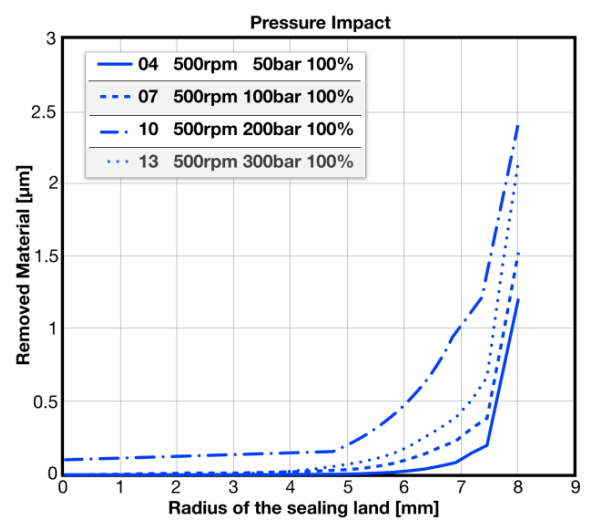

Figure 17: Impact of the Pressure on the Wear Profile

To summarize it can be said, that underbalanced slippers tend to have more wear at lower speeds, higher pressures and higher displacements. However, the deformation of the parts can inverse some of these trends.

\subsection{Connected operating conditions}

Section 5.1 analyzed the run-in for stationary points one at a time. In reality the pump will experience a series of operating conditions, which will lead to effects not considered in 5.1. In order to be able to compare the simulations to measurements, it is necessary to generate the wear profile as it was created in the measurements. The measurements always started in low operating speeds and then gradually increased pressure then speed. In Figure 18 summarizes the resulting wear profile in exactly such a scenario. As expected combining operating conditions with each other results in different results.

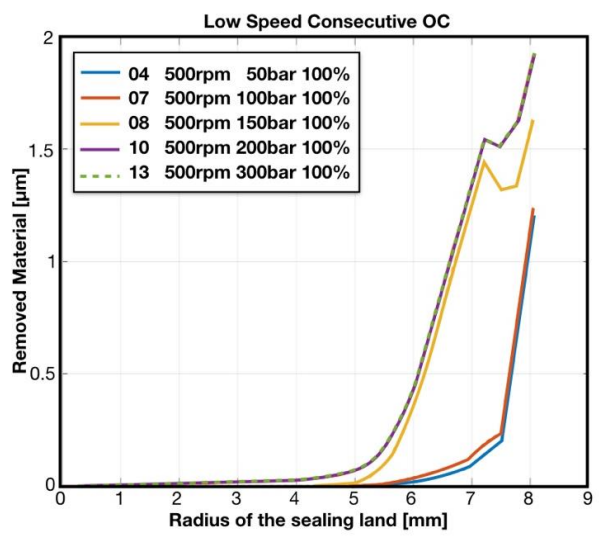

Figure 18: Low speed Wear Profile with Consecutive Operating Conditions

Figure 18 shows the resulting wear as it would occur after the first five operating conditions of the measurement series at $500 \mathrm{rpm}$ and full displacement. The strongest wear would occur at OC $08,500 \mathrm{rpm}, 150 \mathrm{bar}$ and $100 \%$. The wear stopped after $200 \mathrm{bar}$ as indicated by the overlying lines of OC10 and OC13. It should be mentioned at this point, that the numbering of the OC are not in the order they were measured, but rather in an systematic fashion increasing in speed, pressure and displacement.

By comparing Figure 18 and 14 it becomes apparent that OC 08 does not show any wear by itself, however when the wear from $0 \mathrm{C07}$ and OC04 is given as an input, suddenly there is strong wear. In addition, OC13, which showed stronger wear by itself, does not show any sign of wear after all pressure levels until that point have run through the wear-in. This wear-in profile was simulated also with all pressure levels of 
$750 \mathrm{rpm}$, none removed more material. Therefore, it can be concluded that the order of which the run-in occurs does play a role and the resulting shape must not always be the same.

\subsection{Comparison to Measurement}

In order to validate the simulation findings, and to determine if Archard approach yields acceptable results, the simulation results are compared to measurements. Two approaches were taken to compare the results. The wear profile itself and gap height measurements. It should be noted, that the previously presented simulations were performed with the nominal slipper dimensions, taken from the drawings. However, in reality the dimensions vary with the manufacturing tolerances. Therefore, the slippers were measured with a profilometer and the simulations were repeated with the mean, max. and min. of the measured dimensions. Only the mean will be shown in the next section.

\section{Wear profile}

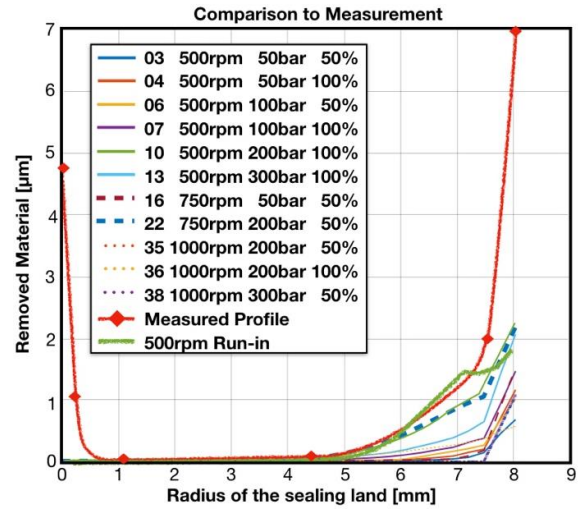

Figure 19: Measured Profile vs Simulated Ones.

The measured wear profile is plotted in Figure 19 , it consists of a series of measured points that were averaged from all slippers. The profile on the outer edge seems to follow the overall trend very well. The outer edge maybe higher worn than in the simulation but one needs to consider that the simulation only considers steady state conditions. It is quite likely that during cold starts or highly dynamic conditions, such as rapid swashing, the slipper tilt increases and wears even more. This might also be the explanation for the wear on the inner edge of the slipper. Only a few simulations indicated wear at the inner radius of the sealing land, but they were on the maximum of the tolerance band and don't explain the wear for the mean dimensions. One explanation could be, that during a cold start, when all temperatures are equally low, the slipper starts to tilt and decrease its fluid film at the outer edge, increase friction and its local temperature. This thermal loading could lead to unusual deformation where the inner sealing land has a lower profile than the outer.

The thick green line represents the accumulated wear profile at low speeds with various pressures (see Figure 18). It suggests that a run-in at low speeds could be sufficient to achieve a working profile for all operating conditions. Overall one could say, that Archard's approach yielded reasonable results, especially for the high wear conditions such as OC10 and $\mathrm{OC} 22$.

\section{Gap Height Measurement}

In order to see if the simulation is capable of predicting the correct performance in terms of power loss and gap height before and after the run-in, the power loss chart will be used again. OC4, $500 \mathrm{rpm}, 50 \mathrm{bar}$ and $100 \%$ displacement is shown in Figure 20.

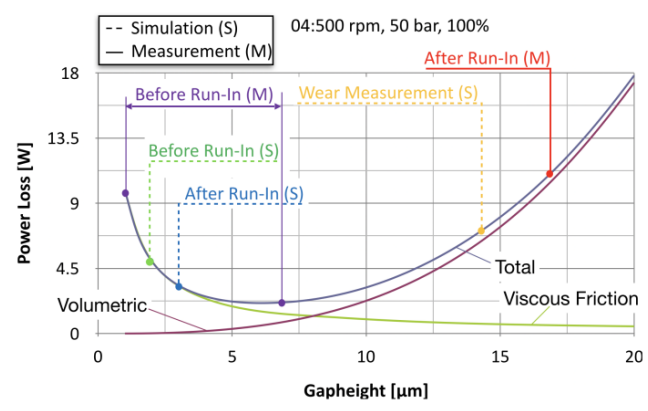

Figure 20: Gap Height Before and After Run-In for Simulation and Measurement at $\mathrm{OC} 4$

The measurement is shown in solid lines, and the simulation in dashed lines. The measured gap heights had a large spread before the run in, this is due to the tolerances of the manufacturing process. After the run-in the gap heights converged, but to a higher value with more losses. The simulation predicts the gap heights of the nominal slipper within the measured tolerance spread. Post run-in both the measured and simulated gap heights increased, however the simulated one does not increase as strongly as the 
measured. This can be explained as follows. For this operating point the simulation predicted some wear, but not very significant. This means that the original shape of the slipper performs sufficiently well. Wear only occurs during low gap heights and therefore high friction. Once the slipper fluid film is sufficient to bear the loads, no more wear occurs. This means wear only occurs when the slipper is left of the optimum point. If the slipper wears off in a different operating point, let's say one with a higher pressure, then the shape changes and it can influence the performance at this lower pressure OC as well. The yellow dotted line shows the simulation output using the measured slipper profile. Using this profile, the simulation predicts a gap height, which is much closer to the measured one.

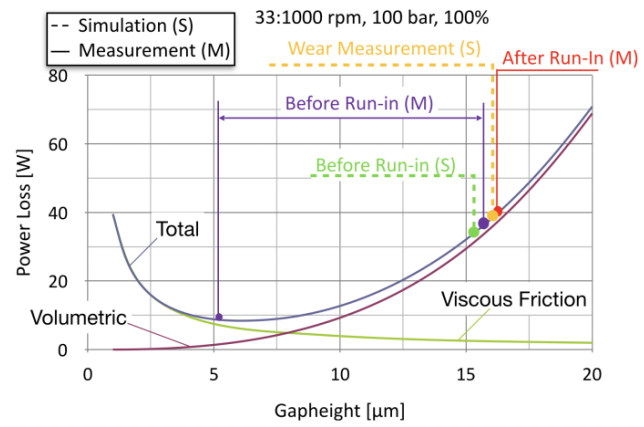

Figure 21: Gap Height Before and After Run-In for Simulation and Measurement at OC33

Figure 21 shows the power loss for OC33. $1000 \mathrm{rpm}, 100 \mathrm{bar}$ and $100 \%$ for the measurement (solid lines) and the simulation before and after run-in. The simulation actually did not predict any wear for this operating condition (before equals after). However, the simulation with the measured profile proves a clear change in gap heights towards higher gap heights and larger losses. These two examples show that the simulation is capable of predicting the resulting performance changes due to the shape change that occurs with wear, however more research is necessary to predict the final shape.

\section{CONCLUSION}

The run-in procedure plays a significant role in today's hydraulic pumps and motors. It allows for wider tolerances, as the incorrect shape of the slipper will be "corrected" by the wear process.
However, it was shown that this final shape must not yield the best design, but rather a design that works in all operating conditions. The goal of this research is to improve the simulation tool Caspar FSTI, by adding a wear-in loop. This loop detects operating conditions that are insufficiently borne due to an insufficient fluid film and calculates the amount of material that needs to be removed. Currently the wear model is based on Archard's law, with an empirically determined wear coefficient. Future work aims to compare this approach with a more complex Fleischer model.

The simulation results are very promising, as it was capable of identifying operating conditions with wear and predicting realistic trends both in shape and magnitude of the wear. In addition, the slipper gap heights before and after run-in were correctly projected as confirmed by gap height measurements.

The wear-in process is not due to a single operating point, neither by extreme conditions at the border of the operating range, but rather consist of a mixture of operating points that occur after each other, each contributing a small part to the final profile shape.

In the next steps a tolerance analysis will be performed to determine the impact the manufacturing tolerances have on the wear process. Furthermore, the impact of the order of the operating points during the run-in will be studied, to answer the question if there is indeed an ideal run-in scenario. Finally, the difference between Archard's and Fleischer's wear model will be investigated.

\section{NOMENCLATURE}

$\begin{array}{ll}T D C & \text { Top Dead Center } \\ F S T I & \text { Fluid Structure Thermal Interaction } \\ S X & \text { Sensor X } \\ c c & \text { Cubic Centimeter per revolution } \\ O C & \text { Operating Condition } \\ N & \text { Normal Force } \\ S & \text { Wear length } \\ H & \text { Hardness } \\ W & \text { Work } \\ k & \text { Wear coefficient } \\ p & \text { Pressure } \\ \rho & \text { Density } \\ \mu & \text { Dynamic viscosity } \\ h_{G} & \text { Gap height } \\ H P & \text { High pressure } \\ L P & \text { Low pressure } \\ \omega & \text { Rotational speed } \\ F_{T G} & \text { Frictional force of the slippers }\end{array}$




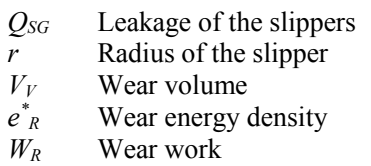

\section{REFERENCES}

[1] Ivantysyn, Roman ; Shorbagy, Ahmed ; et. al.: Analysis of the Run-in Behavior of Axial Piston Pumps. In: IEEE GFPS 2018. Samara, Russia, 2018 - ISBN 9781538647851

[2] Schenk, Andrew: Predicting Lubrication Performance Between the Slipper and Swashplate in Axial Piston Hydraulic Machines, Dissertation, Purdue University, 2014

[3] Ivantysyn, Roman ; Weber, Jürgen: Investigation of the Thermal Behaviour in the Lubricating Gap of an Axial Piston Pump with Respect to Lifetime. In: 11. IFK 2018, 2018

[4] Pelosi, Matteo: An Investigation on the FluidStructure Interaction of Piston/Cylinder Interface, Dissertation, Purdue University, 2012

[5] Ivantysynova, Monika ; Baker, Jonathan: Power loss in the lubricating gap between cylinder block and valve plate of swash plate type axial piston machines. In: International Journal of Fluid Power Bd. 10 (2009), Nr. 2, S. 29-43

[6] Ivantysyn, Roman ; Shorbagy, Ahmed; u. a.: An Approach to Visualize Lifetime Limiting Factors in the Cylinder block / Valve Plate Gap in Axial Piston Pumps. In: FPMC2017-4327, 2017, S. 112

[7] Ivantysyn, Roman ; Weber, Jürgen: "Transparent Pump" - An approach to visualize lifetime limiting factors in axial piston pumps. In: ASME 2016 9th FPNI Ph.D Symposium on Fluid Power. Florianapolis, Brazil, 2016

[8] Shorbagy, A., Ivantysyn, R., Weber, J. An experimental approach to simultaneously measure the temperature field and fluid film thickness in the cylinder block/valve plate gap of an axial piston pump. 9th International Symposium On Turbulence, Heat and Mass Transfer, Rio de Janeiro, Brazil, 2018

[9] Wieczorek, U. and Ivantysynova, M. 2002. Computer Aided Optimization of Bearing and Sealing Gaps in Hydrostatic Machines - The Simulation Tool CASPAR. International Journal of Fluid Power, Vol. 3 (2002), No.1

[10] Ivantysyn, J., and Ivantysynova, M. "Hydrostatic pumps and motors." Academia Book International, New Delhi, India (2001).

[11] Deeken, M. (2004). Simulation of the tribological contacts in an axial piston machine. In IMECE04 (pp. 1-5).

[12] Wegner, D. S., \& Löschner, F. (2016). Validation of the physical effect implementation in a simulation model for the cylinder block / valve plate contact supported by experimental investigations. In IFK2016

[13] Dowd, J. R. and Barwell, F. T. 1974. Tribological interaction between piston and cylinder of a model high pressure pump. Transactions ASLE, 18, pp. 21-30.

[14] Ramalho, A.; Miranda, J.: The relationship between wear and dissipated energy in sliding systems. In: Wear (2006), 260., 361-367

[15] Amiri, M.; Khonsari, M.: On the Thermodynamics of Friction and Wear-A Review. In: Entropy (2010), 12.,1021-1049

[16] Amiri, M.; Khonsari, M. et al.: On the Relationship Between Wear and Thermal Response in Sliding Systems. In: Tribology Letters (2010),38., 147-154

[17] Fleischer, G. et al.: Verschleiß und Zuverlässigkeit, 1980

[18] Bushlya, Volodymyr, et al. "Wear mechanisms of uncoated and coated cemented carbide tools in machining lead-free silicon brass." Wear 376 (2017): 143-151. 